FERMILAB-FN-0802-AD

\title{
An Analytical Approach to Understanding Tevatron Integrated Luminosity
}

\author{
M.J. Syphers
}

May 11, 2007

\begin{abstract}
The recent record-setting performance of the Fermilab Tevatron is the culmination of a long series of efforts to optimize the many parameters that go into generating integrated luminosity for the colliding beams experiments. The instantaneous luminosity is a function of the number of particles of each particle species, the physical extent at the collision point of the transverse and longitudinal particle distributions, and the bunch collision frequency. Meanwhile, the integrated luminosity also depends upon the rate at which particles are lost due to collisions or other means, as well as the rate at which the initial store luminosity can be restored after the end - intentional or otherwise - of the previous store. While many numerical computer models already exist that are used to help optimize the performance of the Tevatron complex, here we take an analytical approach in an attempt to illustrate the most fundamental aspects of integrating luminosity in the Tevatron. We find that the essential features, including recent values of the weekly integrated luminosity, can be understood in a transparent way from basic operational parameters such as antiproton stacking rate and beam emittance growth rate in the Tevatron.
\end{abstract}

\section{INTRODUCTION}

We will build up our understanding in stages. First, we look at the condition where a collider uses two beams with equal bunch intensities, and where particles are lost from the accelerator only due to collisions. Next, we allow the two beams under these same conditions to have different average bunch intensities. And lastly, we introduce a beam lifetime which is independent of luminosity (for example, beam growth due to noise sources) to arrive at a more realistic model.

To begin we consider the case where particles of two equally populated, counter-rotating beams of a collider are lost only due to collisions with each other. For such a "perfect store," given enough time, the delivered integrated luminosity $I_{0}$ must be equal to the number of particles "consumed" divided by the interaction cross section; the luminosity delivered to each experiment would be this number divided by the number of experiments. For a collider with equal number of bunches in each beam, and equal bunch populations, the ultimate integrated luminosity for the store delivered to each experiment would be

$$
I_{0}=\frac{N_{\text {total }}}{n \Sigma}=\frac{B N_{0}}{n \Sigma},
$$


where $B$ is the number of bunches per beam, $N_{0}$ is the initial number of particles per bunch, $n$ is the number of interaction points, and $\Sigma$ is the interaction cross section. This is the maximum total integrated luminosity one could hope for from a single store.

\section{$1.1 \quad$ Equal bunch intensities}

To arrive at the above conclusion analytically, consider the ideal case of a collider with equal bunch populations in each beam, and where the beam size at the interaction point and the bunch length do not change during the store. The luminosity can be written as

$$
\mathcal{L}=\frac{f_{0} B N^{2}}{4 \pi \sigma^{* 2}} \cdot \mathcal{H}
$$

Here, $\sigma^{*}$ is the transverse beam size (considered to be round) at the interaction point, $f_{0}$ is the revolution frequency, and $\mathcal{H}$ is a form factor taking into account crossing angles, "hour glass " effects, and so on. Furthermore, suppose the rate at which the particles in each beam leave the accelerator is given solely by the particle interaction rate, namely,

$$
B \dot{N}=-\mathcal{L} \Sigma n
$$

Inserting Eq. 2 into Eq. 3 and integrating leads to

$$
\mathcal{L}(t)=\frac{\mathcal{L}_{0}}{\left[1+\left(\frac{n \mathcal{L}_{0} \Sigma}{B N_{0}}\right) t\right]^{2}}
$$

where $\mathcal{L}_{0}$ is the initial luminosity at time $t=0$.

The integrated luminosity from the beginning of the store until time $t=T$ is then

$$
I \equiv \int_{0}^{T} \mathcal{L}(t) d t=\frac{\mathcal{L}_{0} T}{1+\mathcal{L}_{0} T\left(n \Sigma / B N_{0}\right)}=I_{0} \cdot \frac{\mathcal{L}_{0} T / I_{0}}{1+\mathcal{L}_{0} T / I_{0}}
$$

where

$$
I_{0} \equiv \frac{B N_{0}}{n \Sigma}
$$

Thus, assuming the store ends intentionally at time $T>>I_{0} / \mathcal{L}_{0}, I_{0}$ would be the asymptotic integrated luminosity of that store.

As an illustration, suppose we have 36 bunches in each beam (as in the Tevatron), $B \cdot N_{0}=$ $250 \times 10^{10}$ particles in each beam (typical number of antiprotons in today's Tevatron operation), and use an inelastic cross section of $60 \mathrm{mb}$. Then, if left to collide "long enough," such a store would produce $I_{0}=21 \mathrm{pb}^{-1}$ of integrated luminosity in each of two detectors. Of course, the time it would take to get to a fraction $f$ of this value would be

$$
T_{f}=\frac{I_{0}}{\mathcal{L}_{0}} \frac{f}{1-f} .
$$

To reach $85 \%$ of $I_{0}$ in our example above, starting with an initial luminosity of $10^{32} \mathrm{~cm}^{-2} \mathrm{sec}^{-1}=$ $0.36 \mathrm{pb}^{-1} / \mathrm{hr}$, say, the store would need to last about two weeks. To reach $95 \%$ of $I_{0}$ would take about a month and a half. 


\subsection{Unequal bunch intensities}

Of course the proton beam in the Tevatron is more intense than the antiproton beam, by a factor of 3-4. Thus, the initial luminosity will be that much higher, and the time to integrate proportionately less. To see this more rigorously, let the population of antiprotons per bunch be $N_{2}$ and that of the protons $N_{1}>N_{2}$, so that the luminosity is

$$
\mathcal{L}=\frac{f_{0} B N_{1} N_{2}}{4 \pi \sigma^{* 2}} \cdot \mathcal{H}
$$

Assuming a one-to-one correspondence in the rate at which protons and antiprotons are consumed (i.e. particles are only lost due to collisions), we define $N_{2}(t)=N(t), N_{1}(t)=N(t)+\Delta N$, where, $\Delta N=N_{1}^{0}-N_{2}^{0}$, and

$$
B \dot{N}=-\mathcal{L} \Sigma n
$$

Here, $N_{1}^{0}$ and $N_{2}^{0}$ are the initial bunch intensities of each species at the beginning of the store. As before, substituting Eq. 6 along with the definitions of $N_{1}(t)$ and $N_{2}(t)$ into the above differential equation and integrating, we get

$$
N(t)=\frac{N_{2}^{0} \Delta N}{N_{1}^{0} e^{\Delta N k t}-N_{2}^{0}}
$$

where $k \equiv n \mathcal{L}_{0} \Sigma / B N_{1}^{0} N_{2}^{0}=n f_{0} \mathcal{H} \Sigma / 4 \pi \sigma^{* 2}$. Thus, the luminosity evolves with time according to

$$
\mathcal{L}(t)=\mathcal{L}_{0} \frac{\Delta N^{2} e^{\Delta N k t}}{\left(N_{1}^{0} e^{\Delta N k t}-N_{2}^{0}\right)^{2}}
$$

which at large $t$ can be written as

$$
\mathcal{L}(t) \sim \mathcal{L}_{0}\left(1-\frac{N_{2}^{0}}{N_{1}^{0}}\right)^{2} e^{-\left(1-N_{2}^{0} / N_{1}^{0}\right) \mathcal{L}_{0} t / I_{0}}
$$

with $I_{0}$ being redefined as $B N_{2}^{0} / n \Sigma$. The integrated luminosity, over a time period $T$, is

$$
I \equiv \int_{0}^{T} \mathcal{L}(t) d t=\frac{B N_{2}^{0}}{n \Sigma} \cdot\left(\frac{e^{\Delta N k T}-1}{e^{\Delta N k T}-\frac{N_{2}^{0}}{N_{1}^{0}}}\right) \quad \Longrightarrow \quad I_{0}, \quad \text { as } t \rightarrow \infty
$$

As one should expect, the resulting asymptotic integrated luminosity is similar to our last result, but here the total number of particles used is that of the less intense beam. (Once the less intense beam is depleted, no more luminosity!)

The time to integrate to a fraction $f$ of the limiting value $I_{0}$ can also be straightforwardly dervied to be

$$
T_{f}=\frac{I_{0} / \mathcal{L}_{0}}{\left(1-N_{2}^{0} / N_{1}^{0}\right)} \ln \left(\frac{1-f N_{2}^{0} / N_{1}^{0}}{1-f}\right),
$$

at which time the luminosity would be reduced by a factor of

$$
\mathcal{L}\left(T_{f}\right) / \mathcal{L}_{0}=(1-f)\left(1-f N_{2}^{0} / N_{1}^{0}\right) .
$$

Take $N_{1}^{0}=2.5 \times 10^{11}$ for the proton beam, and $N_{2}^{0}=7 \times 10^{10}$ for the antiproton beam. We'll use $\mathcal{H}=0.6, f_{0}=47.7 \mathrm{kHz}$, and $\sigma^{*}=25 \mu \mathrm{m}$ as typical operational parameters for the Tevatron. 

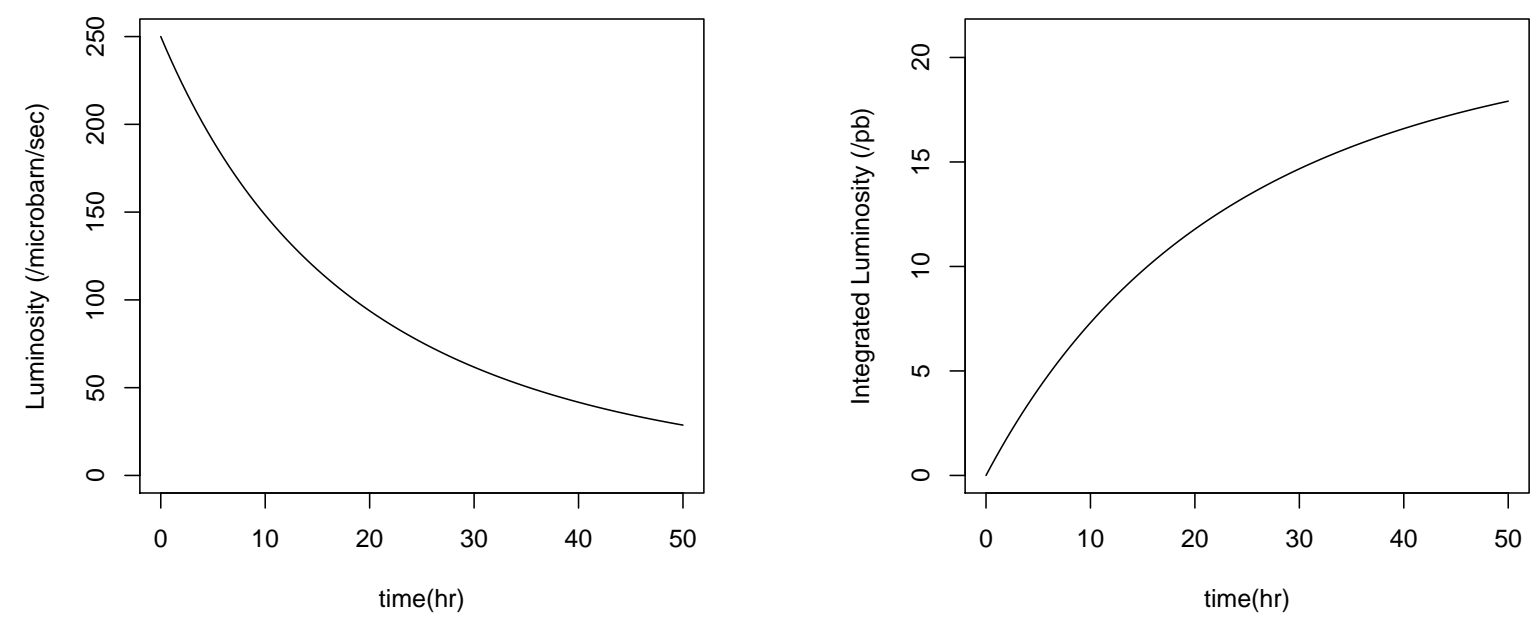

Figure 1: Instantaneous (left) and integrated (right) luminosity vs. time through a "perfect" store, using parameters above. Here, the number of particles in one beam is $\sim 30 \%$ that of the other beam.

Suppose an average store lasts long enough to yield $85 \%$ of the maximum $I_{0}$; note, that the final luminosity would be roughly $10 \%$ of the initial luminosity under these conditions. Then for these parameters,

$$
\begin{aligned}
\mathcal{L}_{0} & \approx 250 \times 10^{30} / \mathrm{cm}^{2} / \mathrm{sec}=250 \mu \mathrm{b}^{-1} / \mathrm{sec}=0.9 \mathrm{pb}^{-1} / \mathrm{hr} \\
T_{f} & \approx 53 \mathrm{hr} \\
I_{0} & \approx 21 \mathrm{pb}^{-1} / \text { store } \\
I_{f}=I_{0.85} & \approx 18 \mathrm{pb}^{-1} / \text { store }
\end{aligned}
$$

Plots of instantaneous and integrated luminosity for a single store under these conditions are provided in Figure 1. Thus, while these store conditions also begin with a total of $250 \times 10^{10}$ antiprotons, the larger number of protons allows the luminosity to integrate to $85 \%$ of its ideal value in about two days rather than 2 weeks. 


\section{SOME WORDS ABOUT PARTICLE LIFETIME}

At the pace depicted in the previous example, one would expect in the Tevatron to integrate about $60 / \mathrm{pb}$ in about 3.5 stores per week at each detector. The maximum integrated luminosity in a week thus far during Tevatron Run II has been about 50/pb, however this was accomplished with 6-7 stores. What is missing in the above analysis is the fact that particles are being lost in the accelerator by mechanisms other than beam-beam collisions at the interaction points. Detailed numerical models are often generated to depict when, where, and how particles are lost around the Tevatron. Typical mechanisms are scattering events (Coulomb and nuclear scattering, for example) as the beam interacts with the residual gas in the vacuum chamber, noise sources in the power supply and radio frequency acceleration systems, and so on. While many such mechanisms are subtle and interesting to accelerator physicists, the general characteristic is that particle oscillation amplitudes grow due to a collection of diffusion processes, and particles can exit at the "aperture" (physical or dynamic) of the accelerator. In a typical Tevatron store, by the time the beam conditions have reached equilibrium and the detectors begin to record data, the aperture is generally determined by the beam collimation system, which is originally set at about 3 times the rms beam size from the beam center in both transverse degrees of freedom. The beam, which starts out roughly Gaussian in transverse dimensions, begins to diffuse due to mechanisms such as those described above, forcing particles into the collimators. If the beam distribution starts out Gaussian, and if particle oscillation amplitudes are altered randomly, then eventually the beam distribution will reach an equilibrium with a maximum emittance which is determined by the aperture. The details of this process can be complex and nonlinear. For our purposes we will model this as a simple noise source generating a constant rate of single particle emittance growth.

Let's use the usual Fermilab definition of normalized 95\% emittance for a transverse coordinate, $x$, namely $\epsilon \equiv 6 \pi \gamma\left\langle x^{2}\right\rangle / \beta$, where $\gamma$ is the Lorentz factor, and the angle brackets denote averaging over the distribution of particle displacements $x$ at a location where the amplitude function has value $\beta$. (Rather than expressing emittance as a "phase space area," we here define it as the second moment of a distribution; this is what is actually reported by the accelerator controls system, and thus familiar quantities can be used in our analyses below.) If this degree-of-freedom is limited by an aperture at a distance $a$ from the beam center, then the equilibrium emittance will be $\hat{\epsilon} \approx 0.92 \pi \gamma a^{2} / \beta$. Next, let's say that in the absence of an aperture, the beam emittance grows at a rate of $\dot{\epsilon}=(6 \pi \gamma / \beta) \cdot d\left\langle x^{2}\right\rangle / d t$. Then the asymptotic lifetime due to diffusion will be $\tau=2 a^{2} /\left(\lambda_{1}^{2} d\left\langle x^{2}\right\rangle / d t\right) \approx 2 \hat{\epsilon} / \dot{\epsilon}$, where $\lambda_{1}$ is the first zero of the Bessel function $J_{0}(x)$. [1] For example, typical emittance growth rates measured in the Tevatron are on the scale of $\dot{\epsilon} \approx 1 \pi \mathrm{mm}-\mathrm{mrad} / \mathrm{hr}$. If the equilibrium maximum emittance were $12 \pi \mathrm{mm}-\mathrm{mrad}$, then the particle lifetime due to diffusion would be about 24 hours. Figure 2 shows the development of the beam intensity over time for these values of $\hat{\epsilon}$ and $\dot{\epsilon}$, when the aperture is at a distance of about 3 times the initial rms beam size. The equilibrium lifetime is acquired almost immediately. In what follows we will assume that a diffusion process (or processes) in conjunction with luminosity contribute to the rate of particle loss in the accelerator, and hence the evolution of beam intensity and luminosity over time can be treated in a straightforward analytical fashion as we see in the next section. ${ }^{1}$

\footnotetext{
${ }^{1}$ Recently, measures have been taken which reduce the effects of beam-beam interactions in the Tevatron. In the presence of beam-beam, the lifetime can often be correlated with luminosity; nowadays, this correlation is much reduced, making our analysis more applicable than before.
} 


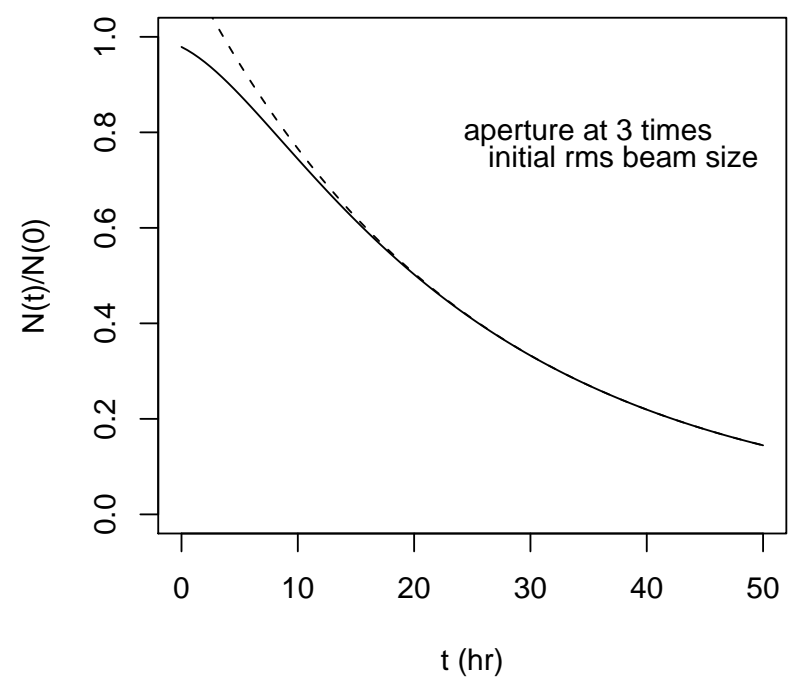

Figure 2: Example of the development of beam intensity over time, due to diffusion. The distance to the limiting aperture from the beam center is initially 3 times the rms beam size, assumed Gaussian at $t=0$. The dotted curve is for exponential decay with lifetime $\tau=24 \mathrm{hr}$.

\section{DIFFERENTIAL EQUATIONS FOR BUNCH INTENSITES}

Our new simplified model will assert that the two beams are round at collision, with identical equilibrium emittances (or, if different emittances, we will assume a single "effective" emittance, $\epsilon=\sqrt{\epsilon_{1} \epsilon_{2}}$ ), and that the diffusion rate of each of the two beams generates an effective emittance growth rate, $\dot{\epsilon}$, which is common to both beams. Akin to Eq. 3, the equations for the bunch population for each of the two beam species will be

$$
\begin{aligned}
& \dot{N}_{1}=-\mathcal{L} \cdot \Sigma \cdot n / B-\frac{1}{\tau} N_{1}=-k N_{1} N_{2}-\frac{1}{\tau} N_{1} \\
& \dot{N}_{2}=-\mathcal{L} \cdot \Sigma \cdot n / B-\frac{1}{\tau} N_{2}=-k N_{1} N_{2}-\frac{1}{\tau} N_{2}
\end{aligned}
$$

where, again, $k=\mathcal{L}_{0} \Sigma n /\left(B N_{1}^{0} N_{2}^{0}\right)=\mathcal{L}_{0} /\left(I_{0} N_{1}^{0}\right)$. Subtracting the two equations and integrating, we find that

$$
N_{1}(t)-N_{2}(t)=\left(N_{1}^{0}-N_{2}^{0}\right) e^{-t / \tau}
$$

which says that if we solve for either $N_{1}(t)$ or $N_{2}(t)$ we can find the other by subtracting or adding the right-hand exponential function in the above equation to our answer. So, we let $N(t) \equiv N_{2}(t)$ and find that

or,

$$
\dot{N}=-k\left(N+\Delta N e^{-t / \tau}\right) \cdot N-\frac{1}{\tau} N,
$$

$$
\dot{N}+\left(\frac{1}{\tau}+k \Delta N e^{-t / \tau}\right) N+k N^{2}=0
$$


This nonlinear differential equation can be turned into a linear first-order differential equation by setting $Z=1 / N$,

$$
\dot{Z}-\left(\frac{1}{\tau}+k \Delta N e^{-t / \tau}\right) Z-k=0,
$$

and standard methods can therefore be used for its solution. The final result for $N(t)$ in terms of initial conditions is

$$
N(t)=N_{2}(t)=N_{2}^{0} \frac{\Delta N e^{-t / \tau}}{N_{1}^{0} e^{\left(1-e^{-t / \tau}\right) \Delta N k \tau}-N_{2}^{0}} .
$$

This rather formidable result (especially the exponential of the exponential of time in the denominator!) is indeed the solution of Eq. 12 and reduces to our result of the previous section in the limit where $\tau \rightarrow \infty$.

We immediately have our result for $N_{1}(t)$, which when simplified becomes

$$
N_{1}(t)=N_{1}^{0} \frac{\Delta N e^{-t / \tau}}{N_{1}^{0}-N_{2}^{0} e^{-\left(1-e^{-t / \tau}\right) \Delta N k \tau}},
$$

whereby we may write down the luminosity as a function of time:

$$
\mathcal{L}(t)=\mathcal{L}_{0} \frac{\Delta N^{2} e^{-2 t / \tau} e^{-\left(1-e^{-t / \tau}\right) \Delta N k \tau}}{\left(N_{1}^{0}-N_{2}^{0} e^{-\left(1-e^{-t / \tau}\right) \Delta N k \tau}\right)^{2}} .
$$

As before, this reduces to our previous result when $\tau \rightarrow \infty$. When $\tau$ is left finite but the bunch intensities become equal, the result is

$$
\mathcal{L}(t) \longrightarrow \frac{\mathcal{L}_{0}}{\left[N k \tau-(1+N k \tau) e^{t / \tau}\right]^{2}} \quad \text { as } \quad N_{1}^{0} \rightarrow N_{2}^{0}=N
$$

Finally, we can obtain an expression for the integrated luminosity during a store by integrating Eq. 15 to get

$$
I(t)=I_{0}\left[1-\frac{\left(N_{1}^{0}-N_{2}^{0}\right) e^{-t / \tau}}{N_{1}^{0} e^{\left(1-e^{-t / \tau}\right) \Delta N k \tau}-N_{2}^{0}}-\frac{I_{0}}{\mathcal{L}_{0} \tau} \frac{N_{1}^{0}}{N_{2}^{0}} \ln \left(\frac{N_{1}^{0}-N_{2}^{0} e^{-\left(1-e^{-t / \tau}\right) \Delta N k \tau}}{N_{1}^{0}-N_{2}^{0}}\right)\right]
$$

which miraculously reduces to our previous result in the event that $\tau \rightarrow \infty$. Note also that for all $t>0$, even as $t \rightarrow \infty, I(t)<I_{0}$ - the second and third terms in Eq. 16 are both negative. That is, a store will never integrate to $I_{0}$ since particles are being lost due to other mechanisms besides collisions at the interaction points.

For completeness, we can also write down the expression for integrated luminosity under the limiting case were $N_{1}^{0}$ approaches $N_{2}^{0}=N$ :

$$
I(t) \longrightarrow I_{0}\left\{1-\frac{1}{(1+N k \tau) e^{t / \tau}-N k \tau}-\frac{I_{0}}{\mathcal{L}_{0} \tau} \ln \left[1+N k \tau\left(1-e^{-t / \tau}\right)\right]\right\} \quad \text { as } \quad N_{1}^{0} \rightarrow N_{2}^{0}=N .
$$

Plots of luminosity and integrated luminosity during stores for various values of $\tau$ are shown in Figure 3. In this figure, we use the same parameters as used in the numerical example at the end of Section 1. We see that a non-zero emittance growth rate (i.e. particle loss mechanisms), thus finite $\tau$, reduces the integrated luminosity per store but allows for more stores per week. So long as the antiproton production can keep pace, the total integrated luminosity per week can still be close to the ideal case explored in Section 1. 

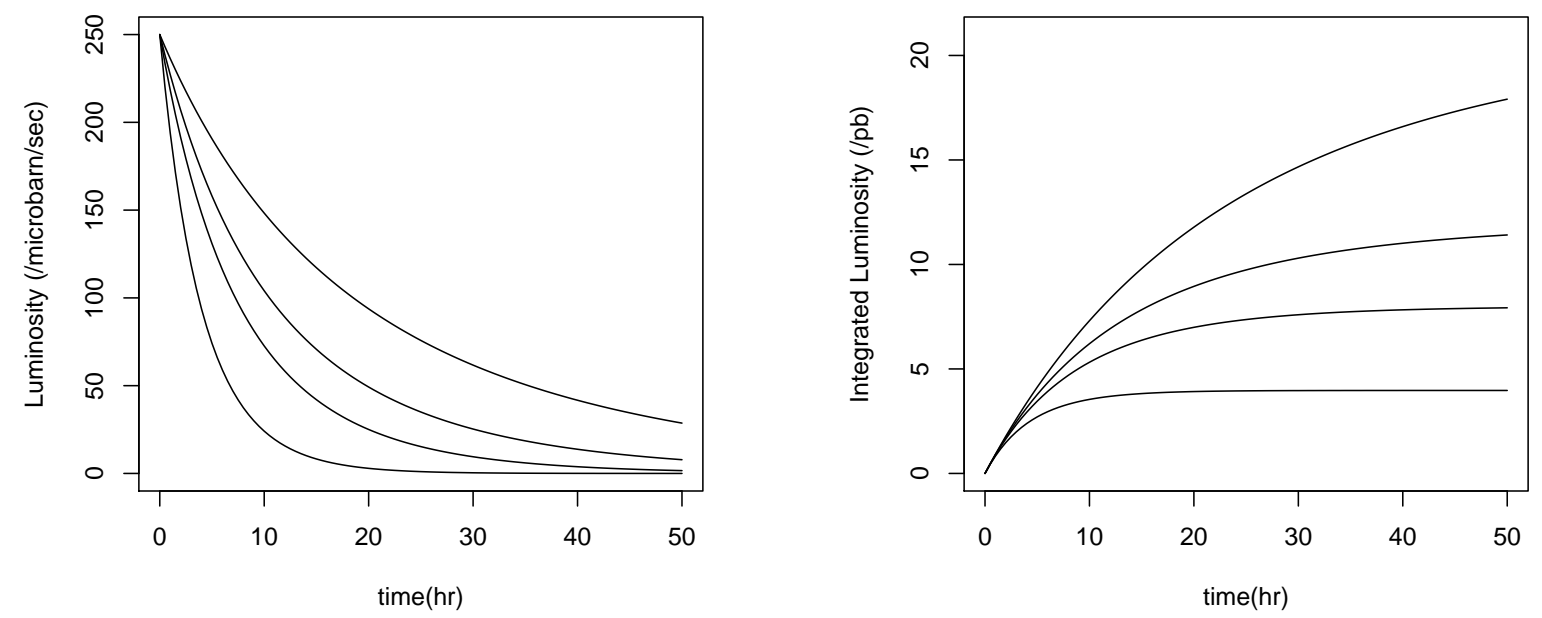

Figure 3: Instantaneous (left) and integrated (right) luminosity vs. time for stores with values of $\tau=10 \mathrm{hr}, 25 \mathrm{hr}, 50 \mathrm{hr}$ and $\tau=\infty$, using same parameter values as in Figure 1. Note that for this set of parameters, indicative of recent Tevatron performance, $I_{0} / \mathcal{L}_{0} \sim 22 \mathrm{hr}$.

\section{APPLICATION TO PRESENT OPERATION}

To look at how well, in an overall sense, our analytical luminosity model works we look at typical average parameters for the Tevatron Run II operation. There have been a few weeks recently during which all stores for the week were ended intentionally. (Often times, stores are ended prematurely due to failures, lightening storms, etc.) For example, take the week of 30 December 2006 through 7 January 2007, which delivered about $45 / \mathrm{pb}$ of integrated luminosity through six stores. During that week, the average initial luminosity was $234 / \mu \mathrm{b} / \mathrm{sec}$ and the average store length was about 25 hours. The average delivered luminosity to each of the two detectors was about $7.5 / \mathrm{pb}$. The average number of antiprotons available for each store was $340 \times 10^{10}$, from which $324 \times 10^{10}$ were sent toward the Tevatron, and $260 \times 10^{10}$ arrived at collision conditions; this yields an average value for $N_{2}^{0}=72.5 \times 10^{9}$. The average number of protons per bunch during this time was $N_{1}^{0}=231 \times 10^{9}$. Average values for other parameters were $\hat{\epsilon}=12 \pi \mathrm{mm}-\mathrm{mrad}$ and $\mathcal{H}=0.59$ at the beginning of a store. A value of $\beta^{*}=30 \mathrm{~cm}$ with these parameters will give the above quoted initial luminosity, and is also consistent with operations.

The typical time between stores ("shot set-up time") was about two hours. To produce the required $324 \times 10^{10}$ antiprotons during the time between the start of neighboring stores, the average antiproton production rate was about $12 \times 10^{10} / \mathrm{hr}$. Along with the above information, our analytical model has one last free parameter, namely the emittance growth rate, $\dot{\epsilon}$. As mentioned in Section 2 a typical scale for emittance growth rate in the Tevatron is on the order of $1 \pi \mathrm{mm}-\mathrm{mrad} / \mathrm{hr}$. 
Adjusting $\dot{\epsilon}$ to make the integrated luminosity in our model adhere to $7.5 / \mathrm{pb}$ per store, the best value is roughly $\dot{\epsilon}=0.78 \pi \mathrm{mm}-\mathrm{mrad} / \mathrm{hr}$ - again, totally consistent with experience. The result of our analytical model with this set of parameters is plotted in Figure 4 along with the actual integrated luminosity data logged by the accelerator controls system for this week of January.

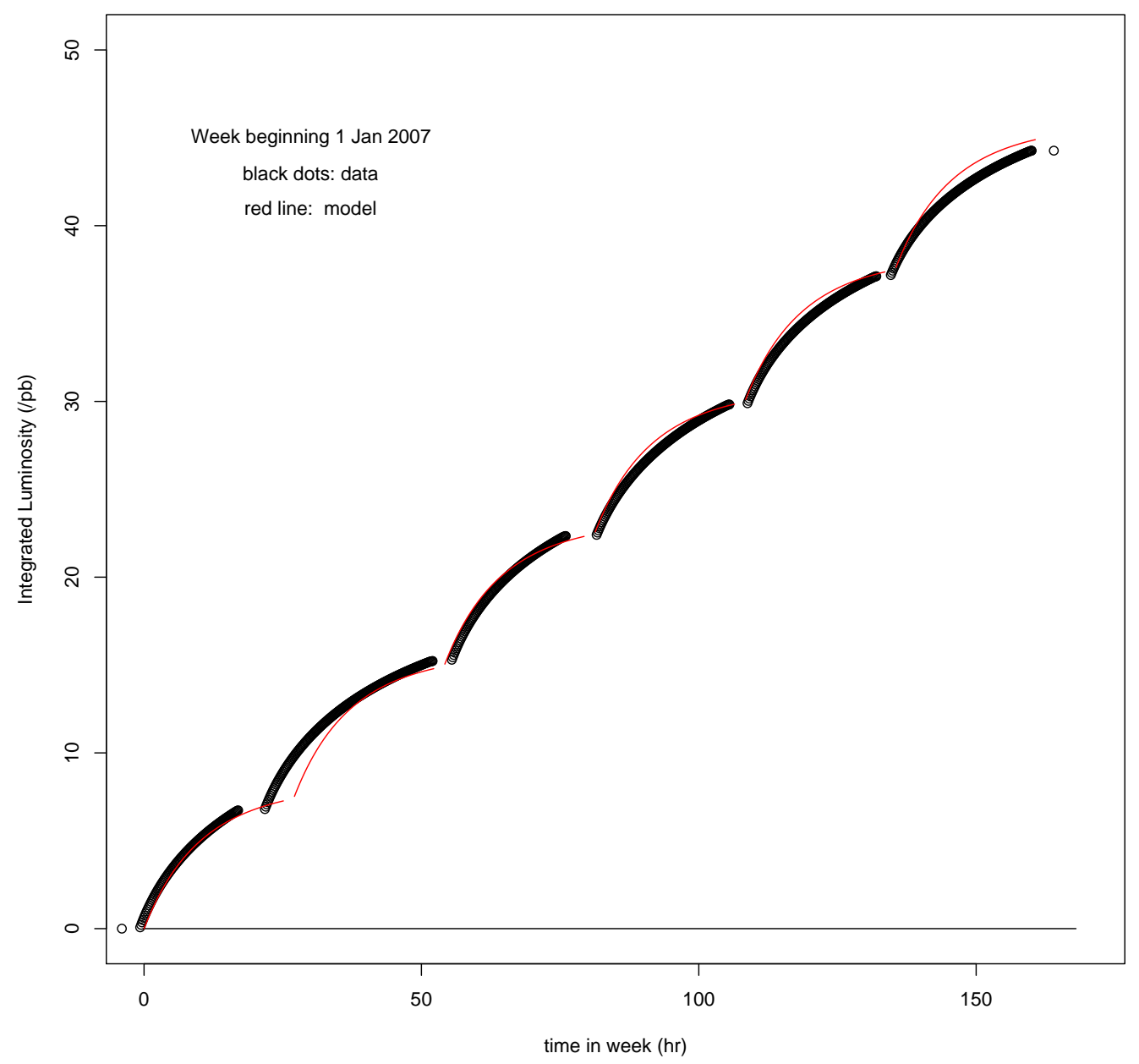

Figure 4: Integrated luminosity for the week 30 December 2006 through 7 January 2007. The large circles are data, while the red (color) curve is the result of our simple analytical model.

We can also see variations in the particle lifetime in each beam throughout the store. If we define particle lifetimes as

$$
\tau_{1} \equiv \frac{N_{1}(t)}{d N_{1}(t) / d t}, \quad \tau_{2} \equiv \frac{N_{2}(t)}{d N_{2}(t) / d t},
$$

then we can take our functional forms, differentiate them, and plot lifetimes vs. time through a 
store. In fact, it is straightforward to see from Equations 9 and 10 that the lifetimes will be

$$
\tau_{1}=\frac{\tau}{1+k \tau N_{2}(t)} \quad \text { and } \quad \tau_{2}=\frac{\tau}{1+k \tau N_{1}(t)}
$$

The results for our typical stores described above are displayed in Figure 5. The lifetimes start out shorter, and evolve into longer lifetimes, and their numerical values - on the scale of 15 to 25 hours - are once again consistent with observations. The higher intensity particle beam typically will have a larger lifetime, as expected, but both will evolve with time as the luminosity is diminished. Given enough time (about 100 hours, in our example), both lifetimes will approach the value of $\tau$.

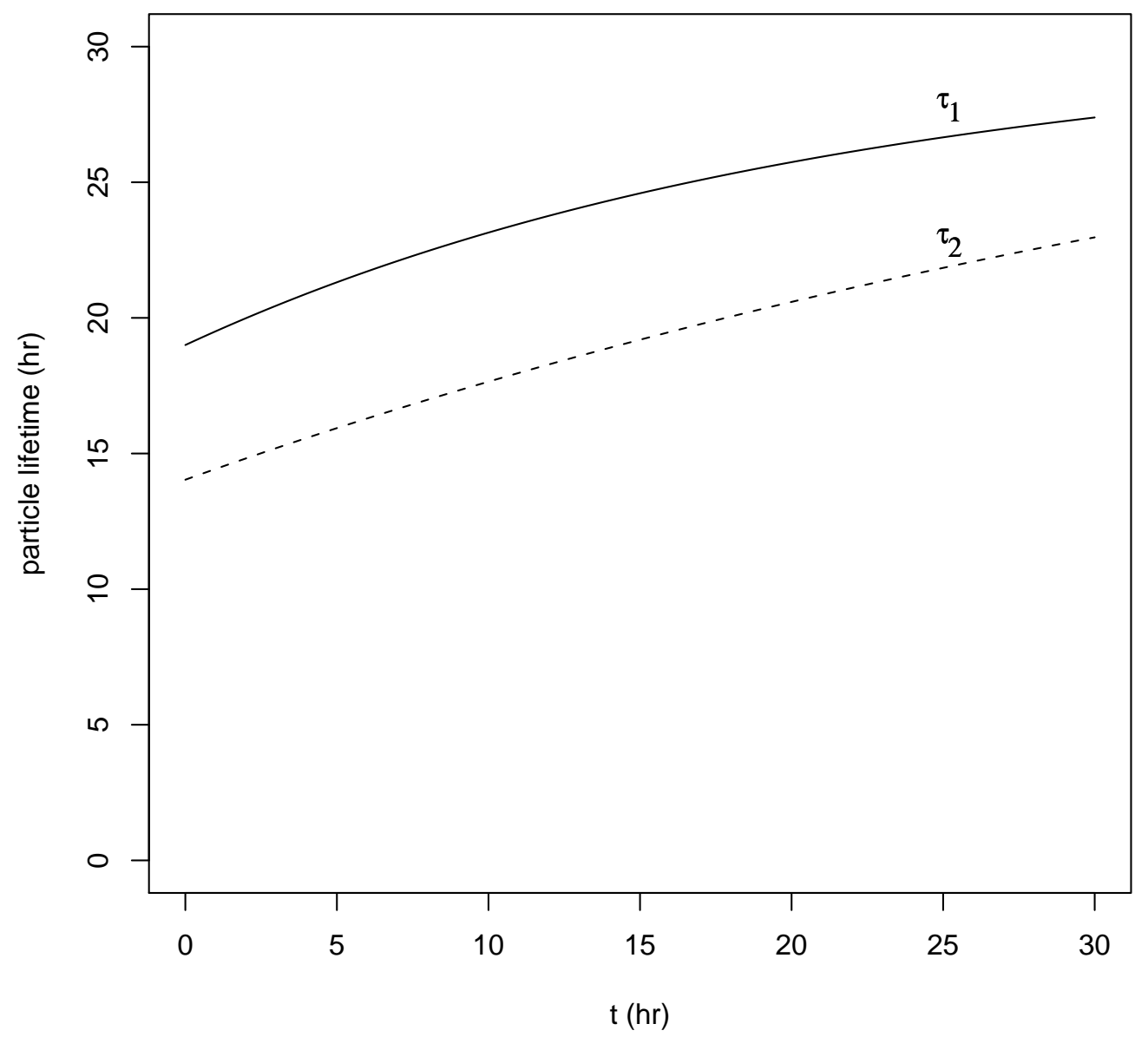

Figure 5: Instantaneous lifetimes of both species of particles through a store, using the typical parameters from our example above. 


\section{STORE LENGTH OPTIMIZATION}

We can quickly examine various features of our analytical model now that a realistic parameter set has been defined. Figure 6 shows the total antiproton intensity throughout a store along with the build-up of antiprotons produced during the store as a function of time. We can now begin

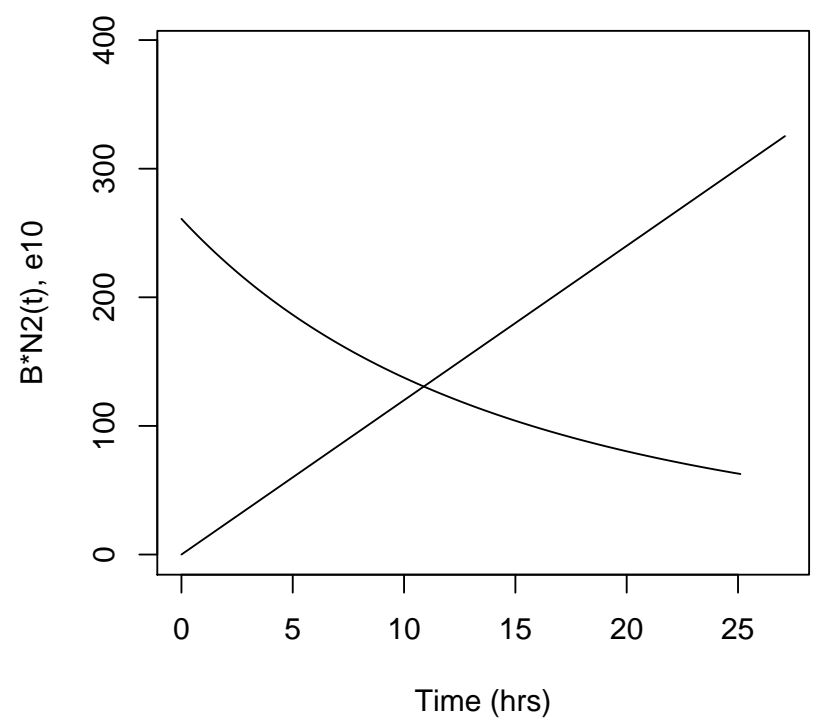

Figure 6: Left: $N_{2}(t)$ during a store and accumulation of $N_{2}$ for the next store. An inefficiency of delivery of antiprotons to collision conditions is built-in.

to use our model to ask questions about the collider operation and to optimize performance. For example, At what stack size does it make sense to end the store and begin a new store? As one might expect, the answer depends upon the antiproton accumulation rate. Suppose we decide to deliver $N_{2}^{0}$ antiprotons to the beginning of a store, and our accumulation rate for antiprotons is $\mathcal{R}$. If we accumulate fast enough, we always have enough antiprotons ready for the next store. If not, then the store must last longer yet, as we saw earlier, there may not be much to gain in integrated luminosity by waiting that long. Thus, there will be an optimal store length for a particular stacking rate, other parameters held constant.

In Figure 7, the antiproton accumulation rate is varied and the average weekly integrated luminosity is plotted as a function of initial number of accumulated antiprotons per store. Again, the scenario of the previous section still holds, where a delivery efficiency $-B \cdot N_{2}^{0}$ at collision over accumulated antiprotons - of $77 \%$ is maintained, etc.. For this figure we assume a store lasts for a time $T$ such that $\mathcal{F} \cdot(\mathcal{R} \cdot T)=B \cdot N_{2}^{0}$, where $\mathcal{F}$ is the delivery efficiency. As can be seen, optimal initial antiproton intensities - and corresponding store lengths - are evident. A plot of optimized 


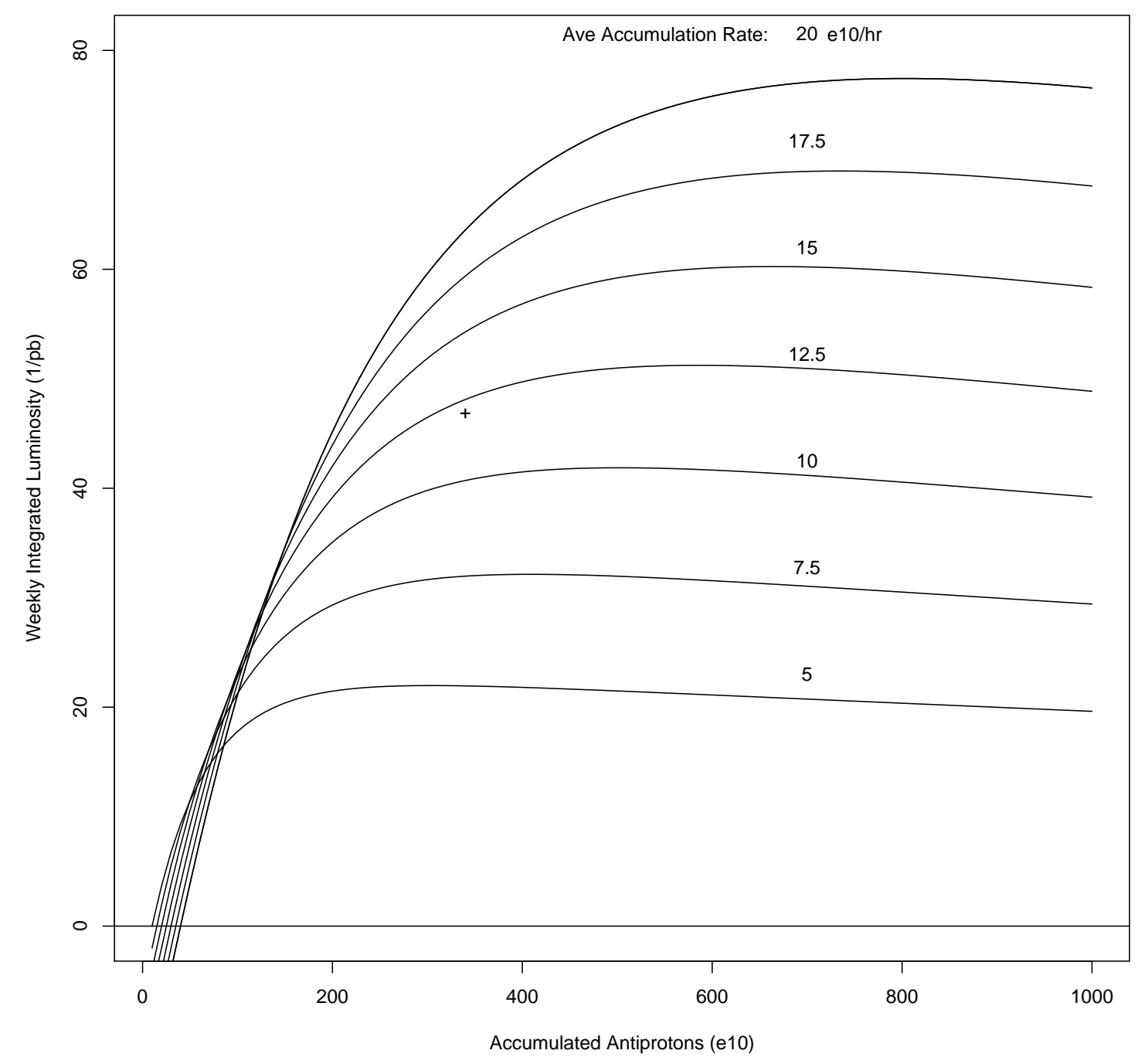

Figure 7: Curves of weekly integrated luminosity vs. number of antiprotons accumulated during a store for given values of the accumulation rate, $\mathcal{R}$. The "+" symbol represents the conditions of our on-going example. For this plot, $\tau=2 \times 12 / 0.78 \mathrm{hr}=30 \mathrm{hr}$. 


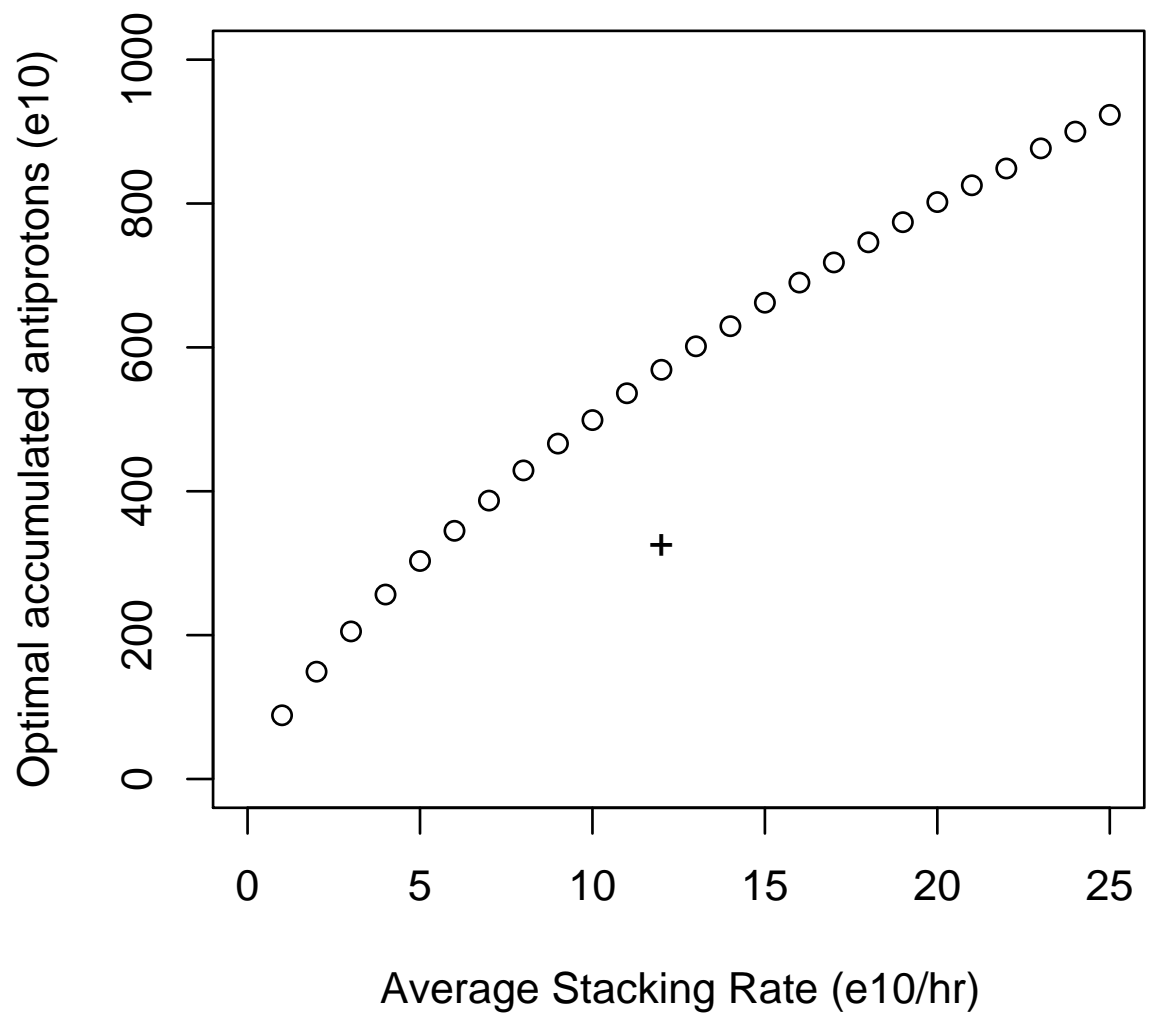

Figure 8: Optimized initial bunch intensities $N_{2}^{0}$ as a function of average accumulation rate, $\mathcal{R}$. The "+" symbol represents the conditions of our on-going example.

initial antiproton intensities vs. accumulation rate is depicted in Figure 8. This figure suggests that if stores could last a bit longer, allowing for higher initial antiproton intensities, then further integrated luminosity could be generated per store. However, looking back at Figure 7, we note that the maximum is rather broad, and the gain in integrated luminosity per week in going from accumulations of $340 \times 10^{10}$ to over $570 \times 10^{10}$ at our present accumulation rate would amount to only a $6 \%$ increase in delivered luminosity.

We can also examine the role of the emittance growth rate in our model on integrated luminosity. Let's stick with our average accumulation rate of $12 \times 10^{10}$ antiprotons per hour, and vary the emittance growth rate in our example. Figure 9 shows curves of weekly integrated luminosity using the same parameters as in Figure 7 . Here, however, we hold the average accumulation rate at $12 \times 10^{10}$ antiprotons per hour, the effective emittance at $12 \pi \mathrm{mm}-\mathrm{mrad}$, and vary the emittance growth rate between 1.5 and $0.25 \pi \mathrm{mm}-\mathrm{mrad} / \mathrm{hr}$, corresponding to $\tau$ between 16 and 96 hours. We 


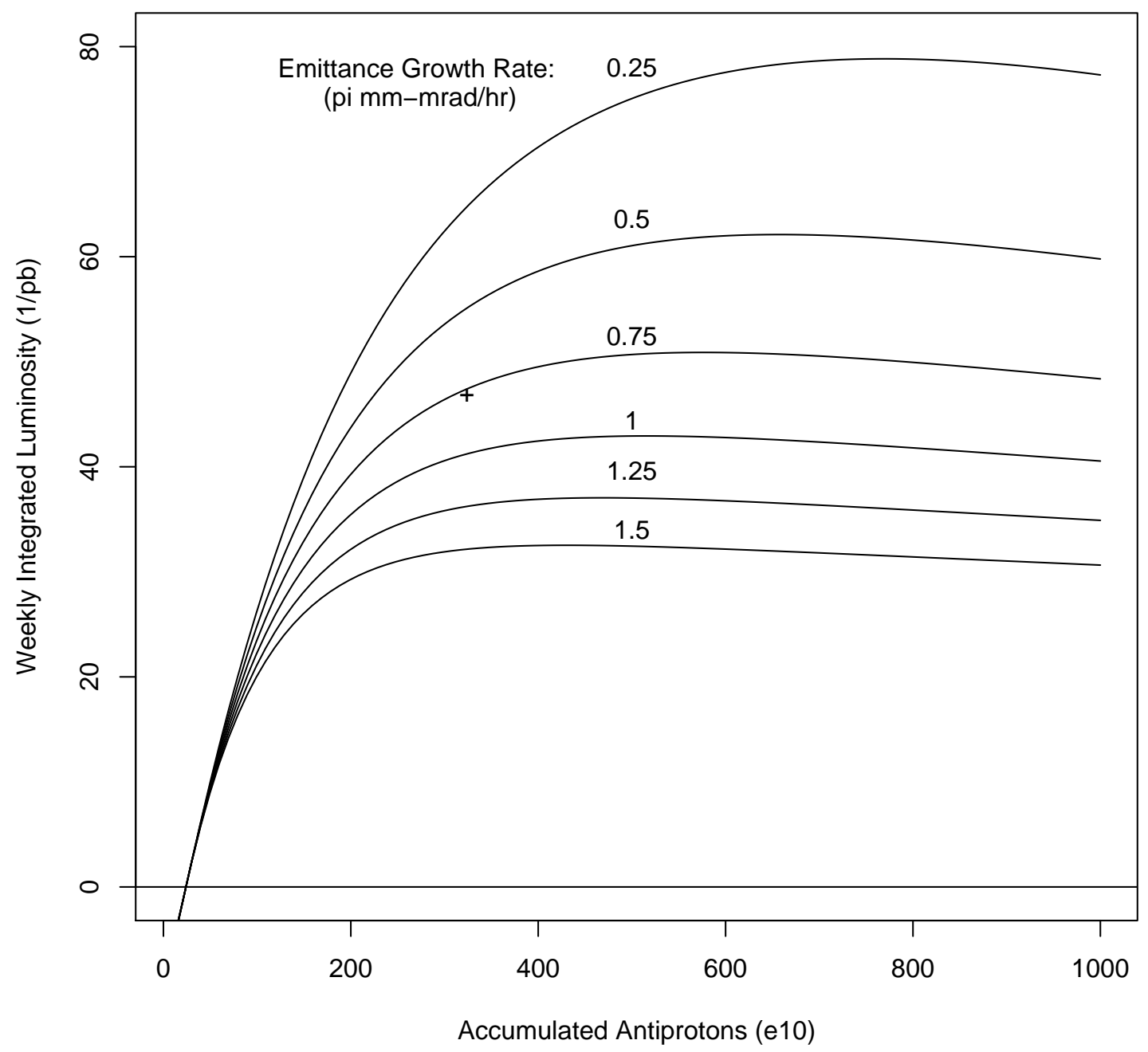

Figure 9: Curves of weekly integrated luminosity vs. number of antiprotons accumulated during a store for given values of effective emittance growth rate, $\dot{\epsilon}$. The "+" symbol represents the conditions of our on-going example from Section 4. The average antiproton accumulation rate $\mathcal{R}=12 \times 10^{10} / \mathrm{hr}$. 
see that for our nominal accumulation rate, if the emittance growth rate were diminished by $33 \%$, for example, then one would contemplate going to higher initial antiproton intensities $-600 \times 10^{10}$, say - and the integrated luminosity could increase by about $33 \%$.

\section{Concluding Remarks}

We have seen that the essential features of a Tevatron store can be described quite well in terms of the conditions setting the initial luminosity, and by the introduction of a particle lifetime, for example generated by an emittance growth rate due to diffusion processes. Several details of actual operation have been left out. For example, the hourglass form factor in the luminosity equation actually develops with time as the bunch length changes during a store (also due to noise sources, as well as due to intrabeam scattering). Also, the beams do influence each other due to beambeam interactions, and so the emittance growth rate, or $\tau$, may depend upon the instantaneous luminosity, for example, and so forth. It is interesting to note, however, that the gross features of Tevatron stores and the integrated luminosity per week can be nicely demonstrated with a simple analytical model which can be used to help sort out important parametric choices to be made during routine operation.

\section{References}

[1] Edwards, D. A., and Syphers, M. J., An Introduction to the Physics of Particle Accelerators, John Wiley, and Sons, New York (1993). Also, see the discussion in M. J. Syphers, "Some Notes on Tevatron Beam Lifetimes and Longitudinal Emittance," Fermilab Internal Report, Beams-doc-1478 (2004). 